Check for updates

Cite this: RSC Adv., 2017, 7, 47565

\title{
A 2 micron passively Q-switched bulk state pulsed laser based on $\mathrm{WS}_{2}$
}

\author{
Xihu Wang, (DD ab Jinlong Xu, ${ }^{c}$ Shufang Gao, ${ }^{d}$ Yunyun Liu, ${ }^{\text {ab }}$ Zhenyu You ${ }^{a}$ \\ and Chaoyang $\mathrm{Tu}^{\star a}$
}

We report a 2 micron passively Q-switched bulk state pulsed laser with a pulse of 430 ns based on graphene-like 2D WS 2 nanosheets. To the best of our knowledge, it is the shortest pulse compared with other bulk state lasers based on $W_{2}$ saturable absorbers at this waveband. The $\mathrm{WS}_{2}$ nanosheets are prepared through a facile hydrothermal reaction. Its max output power and pulse energy are $488 \mathrm{~mW}$ and $5.422 \mu \mathrm{J}$, respectively. The wavelength is distributed from 1934 to $1942 \mathrm{~nm}$. These results reveal that $\mathrm{WS}_{2}$ is a promising nanomaterial in the fields of photonics and electronics.

Received 11th July 2017

Accepted 2nd October 2017

DOI: $10.1039 / \mathrm{c} 7 \mathrm{ra0} 07627 \mathrm{~d}$

rsc.li/rsc-advances

layer consists of a single plane of $\mathrm{W}$ atoms held between two

\section{Introduction}

In recent years, owing to the strong absorption of water molecules, $2 \mu \mathrm{m}$ waveband pulsed lasers have attracted increasing attention because of their vital application in the areas of laser radar systems, medical surgical operations, and environmental protection. Passively Q-switching technology based on saturable absorbers (SAs) has been demonstrated to be a facile and efficient method to realize nanosecond pulsed lasers. ${ }^{1-10}$ It is quite natural that the SA plays a significant role in this process.

Transition element-doped host materials ${ }^{1-3}$ and semiconductor saturable absorber mirrors $\left(\right.$ SESAMs $^{4,5}$ are the important traditional SA materials. However, the development of traditional SA materials is hampered by their narrow absorption band, complex manufacturing processes and high cost. Fortunately, as a novel SA material, the appearance of graphene brings a new idea to explore more valuable SA materials. ${ }^{11}$ This $2 \mathrm{D}$-structured layered carbon material possesses a Dirac-like gapless electronic band structure, resulting in broadband light absorption. ${ }^{11}$ Besides, due to its simple manufacturing processes and low cost, graphene has drawn much more attention to realize pulsed lasers at broadband. ${ }^{6,12-16}$ Inspired by the 2D structure of graphene, a new family of graphene-like 2D materials, including transition metal dichalcogenides (TMDs), ${ }^{7,17,18}$ topological insulators (TIs) ${ }^{8}$ and black phosphorus (BP), ${ }^{9}$ has been developed to realize pulsed lasers rapidly. As a representative of the family of TMDs, each $\mathrm{WS}_{2}$

${ }^{a}$ Key Laboratory of Optoelectronic Materials Chemistry and Physics of CAS, Fujian Institute of Research on the Structure of Matter, CAS, Fuzhou, Fujian 350002, China.E-mail: tcy@fjirsm.ac.cn

${ }^{b}$ University of Chinese Academy of Sciences, Beijing 100039, China

'School of Electronic Science and Engineering, Collaborative Innovation Center of Advanced Microstructures, Nanjing University, Nanjing 210093, China

${ }^{d}$ School of Physics and Optoelectronic Engineering, Yangtze University, Jingzhou, 434023, PR China planes of S atoms by strong covalent bonds, and these single layers are stacked together by weak Van der Waals forces. ${ }^{19}$ It is interesting that its bandgap shifts from indirect $(1.3 \mathrm{eV})$ for bulk state to direct $(2.1 \mathrm{eV})$ for monolayer flakes. ${ }^{20}$ Hence, its bandgap could be engineered by manipulating the thickness or atomic defects, resulting in broadband saturable absorption directly. ${ }^{18,21-24}$ In 2015, $\mathrm{MaO}^{21}$ demonstrated a stable mode-locked fiber laser operation by depositing $\mathrm{WS}_{2}$ nanosheets on an Er-doped fiber for the first time. Later on, $\operatorname{Tan}^{22}$ realized a $\mathrm{WS}_{2}$-based Q-switched waveguide laser in a Nd:YAG crystal. In addition, Luan $^{23}$ first reported its nonlinear saturable absorption property at around $2 \mu \mathrm{m}$ and realized $660 \mathrm{~ns}$ bulk state laser pulses. In order to have a wide variety of practical applications, producing even shorter pulses with higher laser power is still urgently needed and worthy of further investigation.

In this paper, we demonstrate a Q-switched bulk laser of 430 ns with a $\mathrm{WS}_{2}$ SA material at about $2 \mu \mathrm{m}$. As far as we know, it has the narrowest pulse width compared with those of other 2 $\mu \mathrm{m}$ bulk lasers based on $\mathrm{WS}_{2}$. The $\mathrm{WS}_{2}$ was prepared through a facile hydrothermal reaction. A better quality of $\mathrm{WS}_{2} \mathrm{SA}$ film was prepared by a spin coating technique. Its phase, morphology and absorbance at this waveband were investigated. Our results indicated the $\mathrm{WS}_{2}$ is a type of promising nanomaterial in the fields of photonics and electronics.

\section{Experimental section}

The few layered $\mathrm{WS}_{2}$ was synthesized by a facile one-step hydrothermal reaction method. All of the chemicals used in our experiments were of analytical purity and were used without further purification. In a typical experiment, $0.5116 \mathrm{~g} \mathrm{WCl}_{6}$ and $0.4846 \mathrm{~g} \mathrm{C}_{2} \mathrm{H}_{5} \mathrm{NS}$ were dissolved in $16 \mathrm{~mL}$ deionized water. After violent stirring for $1 \mathrm{~h}$ using a magnetic stirrer at room 


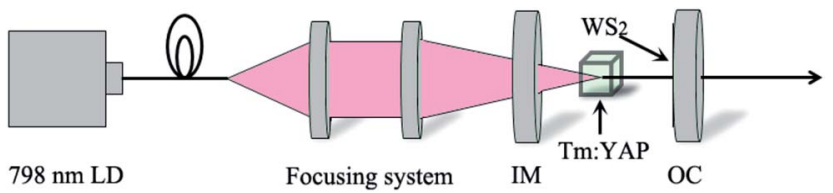

Fig. 1 Schematic of the experimental setup.

temperature, the solution was transferred into a $20 \mathrm{~mL}$ Teflonlined stainless steel autoclave and then heated at $538 \mathrm{~K}$ for $24 \mathrm{~h}$. After cooling naturally, the products were washed with absolute ethyl alcohol three times. Finally, the products were dried in air at $60{ }^{\circ} \mathrm{C}$ for $6 \mathrm{~h}$.

To compress the volume of the laser setup, a compact planeplane straight cavity was chosen in our experiment. Fig. 1 shows the schematic experimental setup. The gain medium was a $3 \times$ $3 \times 5 \mathrm{~mm}^{3}$ a-cut Tm:YAP crystal. It was wrapped in indium foil and mounted in a bronze holder with water-cooling to $293 \mathrm{~K}$. It was end pumped by a fiber-coupled continuous wave diode laser at $798 \mathrm{~nm}$ with a core diameter of $100 \mu \mathrm{m}$. The pump light was then focused into the gain medium by a focusing system. The input mirror (IM) was a plane mirror coated for
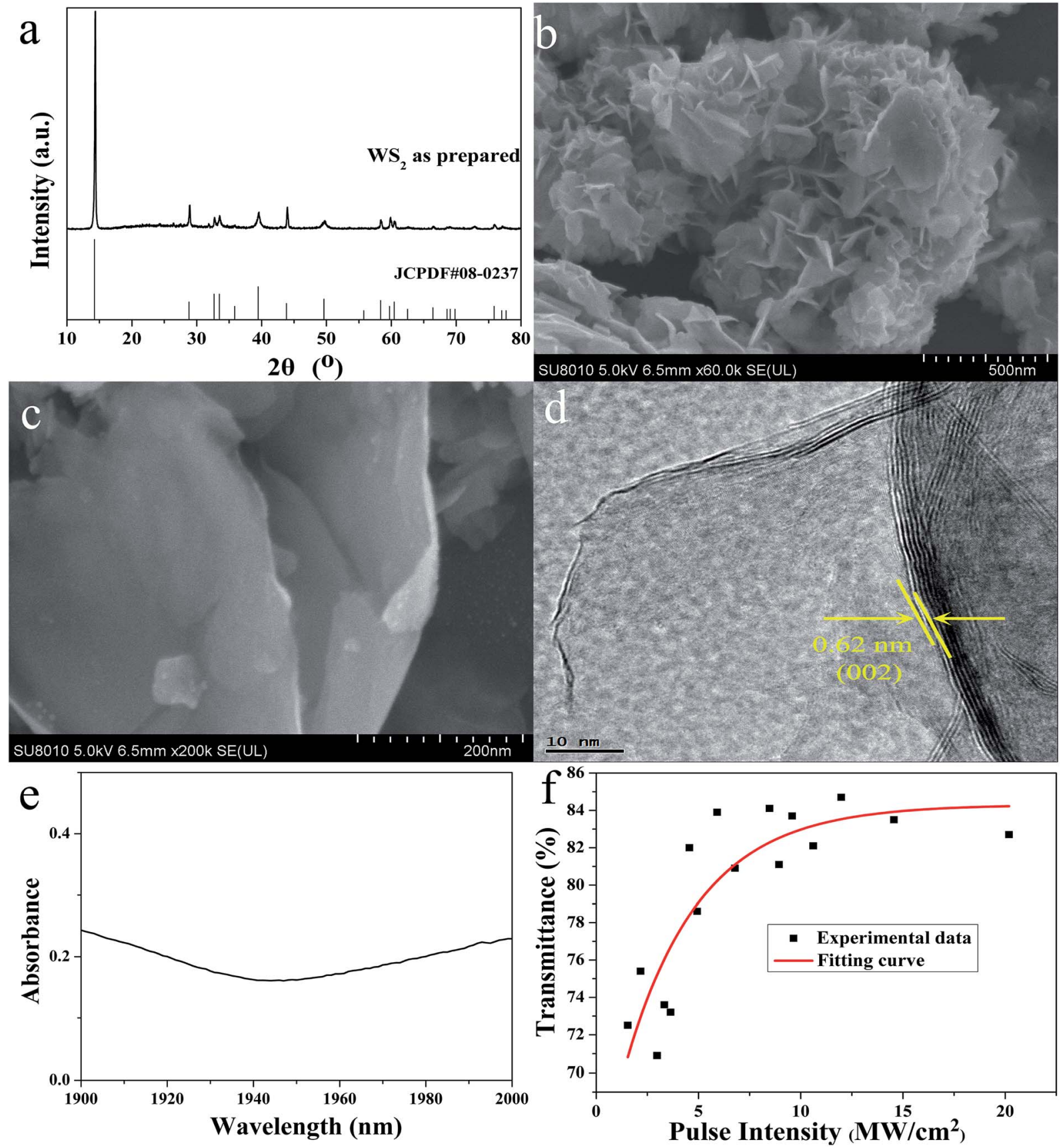

Fig. 2 (a) XRD diffraction pattern, (b and c) SEM images, (d) TEM image, (e) absorption spectrum from 1900 to $2000 \mathrm{~nm}$, and (f) nonlinear transmittance of the $\mathrm{WS}_{2}$ nanosheets. 
antireflection (AR) at around $798 \mathrm{~nm}$ and high-reflection (HR) at around $1980 \mathrm{~nm}$. The output coupler (OC) was also a plane mirror with $3.5 \%$ transmittance at $1980 \mathrm{~nm}$. To reduce the insert loss, the $\mathrm{WS}_{2}$ dispersion was directly spin-coated on the OC.

\section{Results and discussion}

The phase of the products was tested by X-ray powder diffraction (XRD). As indicated in Fig. 2(a), the products display a pure phase of $\mathrm{WS}_{2}$ with a space group $P 6_{3} / m m c$ (194), in good agreement with the standard card JCPDF \#08-0273. To obtain uniform $\mathrm{WS}_{2}$ nanosheets, a certain amount of the products was dispersed in absolute ethyl alcohol and centrifuged at $1500 \mathrm{rpm}$ for $20 \mathrm{~min}$. The morphology of the upper supernatant was studied by scanning electron microscopy (SEM). Fig. 2(b) shows that an abundance of $\mathrm{WS}_{2}$ nanosheets were obtained. Fig. 2(c) was also obtained by SEM with higher resolution. From this sub-micro sized $0 \mathrm{D}$ aggregate, we can clearly ascertain the 2D nature of $\mathrm{WS}_{2}$ and its thickness was measured to be about $5 \mathrm{~nm}$. Fig. 2(d) shows the typical transmission electron microscope image (TEM). It reveals the stacking of the $\mathrm{WS}_{2}$ (002) layers with an interplanar spacing of $0.62 \mathrm{~nm}$. The absorbance characteristics were explored through the infrared spectra. As shown in Fig. 2(e), $\mathrm{WS}_{2}$ possessed broadband absorption characteristics in the wavelength ranging from 1900 to $2000 \mathrm{~nm}$. The absorbance at this waveband was around 0.2 , indicating that the $\mathrm{WS}_{2}$ may be applied as a SA for this waveband. We measured its SA behavior through a pump-probe measurement. To obtain the nonlinear transmission curve, we employed a homemade acousto-optic Q-switched bulk laser using Tm:YAP as the gain medium. The laser power before and after the $\mathrm{WS}_{2}$ was recorded. The corresponding result is shown in Fig. 2(f). By fitting the curve with the equation

$$
T(I)=1-\Delta T \times \exp \left(-I / I_{\mathrm{sat}}\right)-T_{\mathrm{ns}}
$$

where $T(I)$ is the transmittance, $\Delta T$ is the modulation depth, $I$ is the input intensity, $I_{\text {sat }}$ is the saturation intensity, $T_{\mathrm{ns}}$ is the

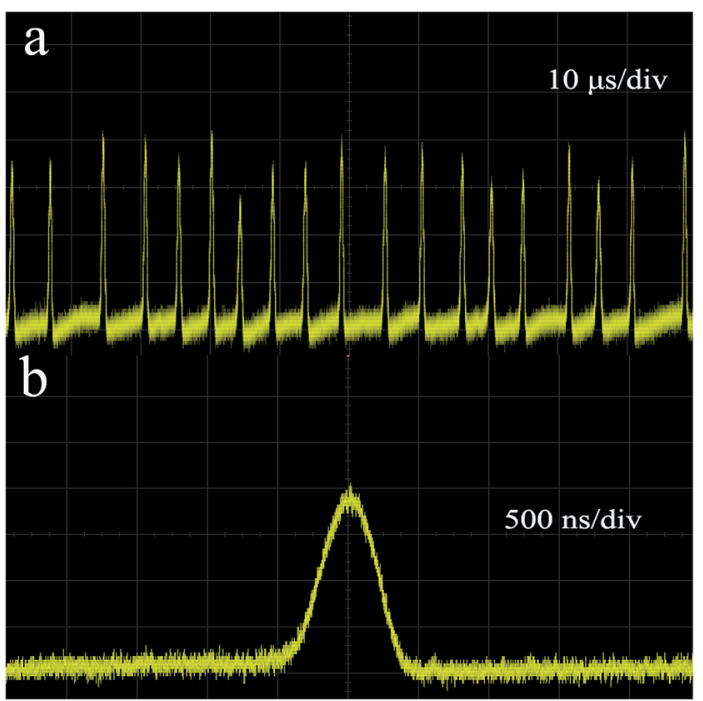

Fig. 4 (a) The Q-switching pulse train and (b) a typical single pulse profile at an absorbed pump power of $5.3 \mathrm{~W}$.

nonsaturable loss, the saturation intensity $I_{\text {sat }}$ and the modulation depth $\Delta T$ are extracted as $3.66 \mathrm{MW} \mathrm{cm}^{-2}$ and $20.56 \%$, respectively. The nonsaturable loss is $15.70 \%$, and considering that the transmittance of the quartz substrate is about $93.50 \%$ at $1.9 \mu \mathrm{m}$, the loss of the $\mathrm{WS}_{2}$ is about $9.20 \%$.

Initially, we investigated the performance of the continuouswave Tm:YAP laser without $\mathrm{WS}_{2}$. When the absorbed pump power was tuned from the threshold to $6 \mathrm{~W}$, no self Q-switching was observed during this course. Then the $\mathrm{WS}_{2}$ dispersion was directly spin-coated onto the OC and it was placed as shown in Fig. 1. Once the absorbed pump power exceeded the threshold of $3.54 \mathrm{~W}$, the stable pulsed laser train began to be realized. Fig. 3(a) shows the dependence of the pulse width and repetition rate on the absorbed pump power. When the absorbed pump power was at the threshold of $3.54 \mathrm{~W}$, the pulse width was at the maximum of $1350 \mathrm{~ns}$. Once the absorbed pump power

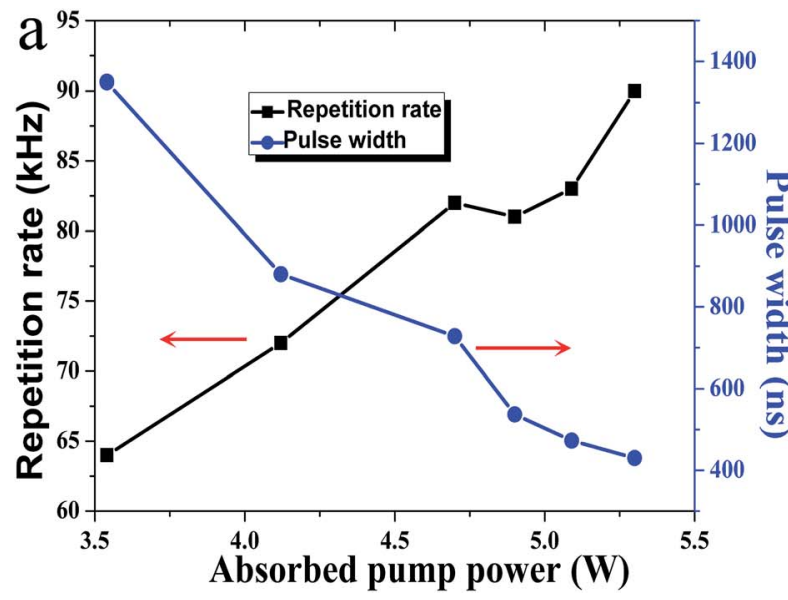

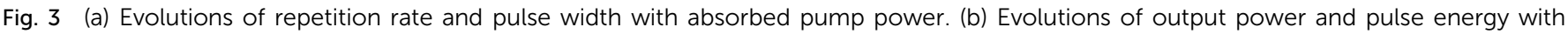
absorbed pump power.

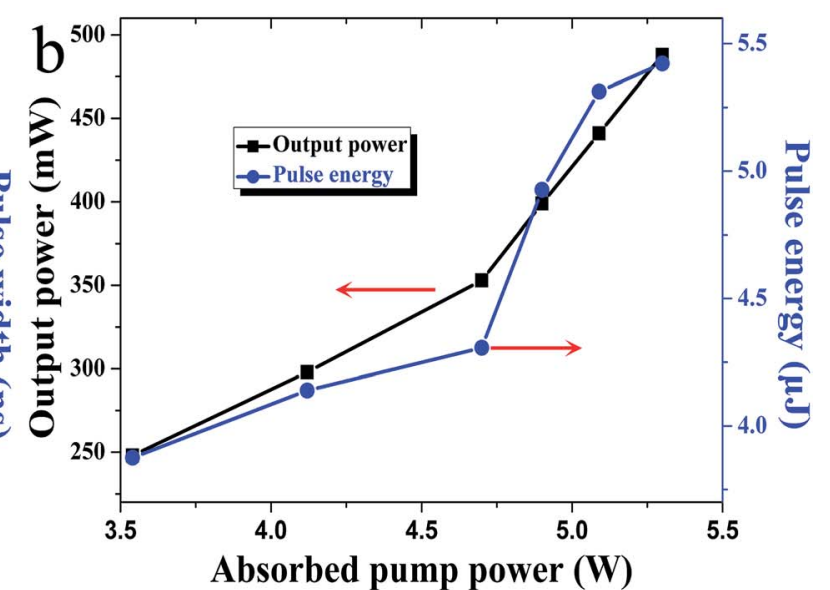




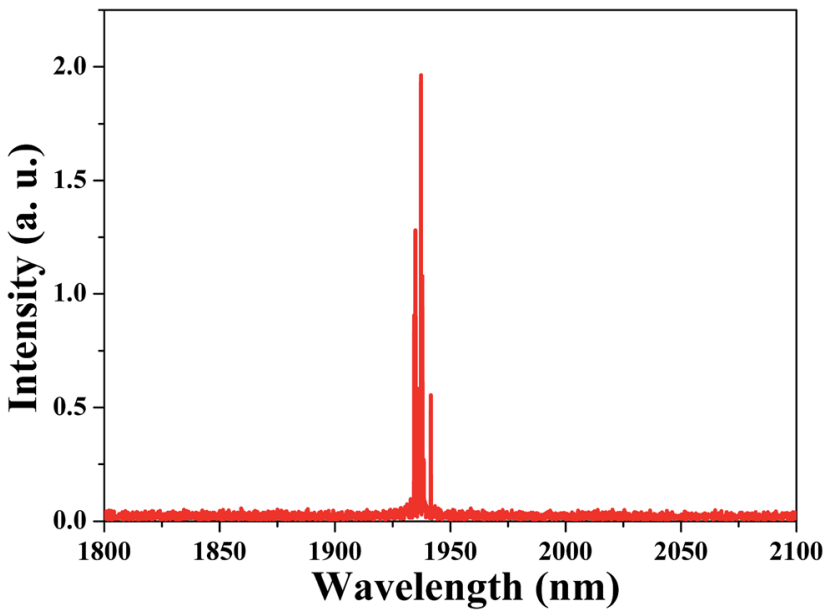

Fig. 5 The laser spectra at an absorbed pump power of $5.3 \mathrm{~W}$.

was increased, the pulse width of the pulse train became continuously narrower. When the absorbed pump power exceeded $5.3 \mathrm{~W}$, the pulse train began to become unstable. The stable pulse train appeared again once the absorbed pump power was below $5.3 \mathrm{~W}$. As a consequence, the $\mathrm{WS}_{2}$ was undamaged at this irradiation intensity. The pulse width reached a minimum of $430 \mathrm{~ns}$ when the absorbed pump power was $5.3 \mathrm{~W}$. As shown in Fig. 3, the repetition rate increased almost linearly from 64 to $90 \mathrm{kHz}$. We also recorded the output power. The output power increased from a minimum of 248 $\mathrm{mW}$ to a maximum of $488 \mathrm{~mW}$. The corresponding result is shown in Fig. 3(b). According to these data, the pulse energy was obtained as shown in Fig. 3(b). A maximum pulse energy of $5.422 \mu \mathrm{J}$ could be deduced.

Fig. 4(a) shows the typical pulse train when the absorbed pump power was $5.3 \mathrm{~W}$. To investigate the stability of Qswitching, we further evaluated the quality of pulse-amplitude equalization. It could be characterized by clock amplitude jitter (CAJ). The CAJ is defined as the ratio of the standard deviation $\sigma$ to the mean value $(M)$ of the intensity histogram at the pulse peak intensity, as described below:

$$
\mathrm{CAJ}=\sigma / M \times 100 \%
$$

The CAJ of the pulse train in Fig. 4(a) was calculated to be $9.54 \%$, revealing good intensity stability. Fig. 4 (b) is a typical

Table 1 Comparison of this $2 \mu \mathrm{m}$ passively $\mathrm{Q}$-switched bulk laser performance with other SAs

\begin{tabular}{lccll}
\hline Type of SA & $\begin{array}{l}\text { Min pulse } \\
\text { width/ns }\end{array}$ & $\begin{array}{l}\text { Max pulse } \\
\text { energy/ } \mathrm{JJ}\end{array}$ & $\begin{array}{l}\text { Repetition } \\
\text { rate/kHz }\end{array}$ & Ref. \\
\hline Graphene & 285 & 1.6 & 190 & 25 \\
Gold nanorod & 796 & 4.9 & 77 & 26 \\
Black phosphorus & 1780 & 7.84 & 19.25 & 27 \\
$\mathrm{MoS}_{2}$ & 800 & 2.08 & 48.09 & 28 \\
$\mathrm{WS}_{2}$ & 660 & 17.1 & 63 & 23 \\
$\mathrm{WS}_{2}$ & 430 & 5.42 & 90 & This work
\end{tabular}

single pulse profile. Simultaneously, the laser spectrum was recorded. As shown in Fig. 5, the wavelength was distributed from 1934 to $1942 \mathrm{~nm}$.

In the end, we compared our results with those of $2 \mu \mathrm{m}$ bulk state lasers with other SAs. As shown in Table 1, we listed the min pulse width, max pulse energy and repetition rate. Based on these data the laser results, especially the min pulse width, could be considered as superior or at least comparable to the others. Even so, our present results may be improved by optimizing the laser cavity and the quality of the SA film. It would be interesting for this to be further developed.

\section{Conclusion}

$2 \mu \mathrm{m}$ passively Q-switched bulk laser operations have been demonstrated using $\mathrm{WS}_{2} \mathrm{SA}$. The min pulse width could be as low as $430 \mathrm{~ns}$. This is the shortest pulse compared with other equivalent $\mathrm{WS}_{2}$ materials in this waveband. Its corresponding output power and pulse energy were $488 \mathrm{~mW}$ and $5.422 \mu \mathrm{J}$, respectively. Our results reveal that this $\mathrm{WS}_{2}$ SA could be a promising SA material to realize $2 \mu \mathrm{m}$ passively Q-switched bulk pulse lasers and that it possesses a potential application in the fields of photonics and electronic.

\section{Conflicts of interest}

There are no conflicts to declare.

\section{Acknowledgements}

This work is supported by the National Nature Science Foundation of China (51472240, 61675204, 11304313 and 11647065); The National Key Research and Development Program of China (2016YFB0701002); the Strategic Priority Research Program of the Chinese Academy of Science (XDB20010200); the State Key Laboratory of Structural Chemistry (20160012); the Science and Technology Plan Cooperation Project of Fujian Province (2015I0007); and the Nature Science Foundation of Fujian Province (2015J05134, 2016J01274).

\section{References}

1 Y. F. Chen, S. W. Tsai and S. C. Wang, High-power diodepumped Q-switched and mode-locked $\mathrm{Nd}$ : $\mathrm{YVO}_{4}$ laser with a $\mathrm{Cr}^{4+}$ : YAG saturable absorber, Opt. Lett., 2000, 25, 1442.

2 H. T. Huang, J. L. He, B. T. Zhang, J. F. Yang, J. L. Xu, C. H. Zuo and X. T. Tao, $\mathrm{V}^{3+}$ : YAG as the saturable absorber for a diode-pumped quasi-three-level dual-wavelength $\mathrm{Nd}$ : GGG laser, Opt. Express, 2010, 18, 3352.

3 V. N. Philippov, A. V. Kir'yanov and S. Unger, Advanced configuration of erbium fiber passively Q-switched laser with $\mathrm{Co}^{2+}$ : ZnSe crystal as saturable absorber, IEEE Photonics Technol. Lett., 2004, 16, 57.

4 R. Fluck, B. Braun, E. Gini, H. Melchior and U. Keller, Passively Q-switched 1.34- $\mu \mathrm{m}$ Nd: YVO4 microchip laser with semiconductor saturable-absorber mirrors, Opt. Lett., 1997, 22, 991. 
5 A. Klenner, M. Golling and U. Keller, High peak power gigahertz Yb:CALGO laser, Opt. Express, 2014, 22, 11884.

6 J. L. Xu, X. L. Li, J. L. He, X. P. Hao, Y. Yang, Y. Z. Wu and B. T. Zhang, Efficient graphene $Q$ switching and mode locking of $1.34 \mu \mathrm{m}$ neodymium lasers, Opt. Lett., 2012, 37, 2652.

7 S. Wang, H. Yu, H. Zhang, A. Wang, M. Zhao, Y. Chen and J. Wang, Broadband Few-Layer $\mathrm{MoS}_{2}$ Saturable Absorbers, Adv. Mater., 2014, 26, 3538.

8 Z. Luo, C. Liu, Y. Huang, D. Wu, J. Wu, H. Xu and J. Weng, Topological-Insulator Passively Q-Switched Double-Clad Fiber Laser at $2 \mu \mathrm{m}$ Wavelength, IEEE J. Sel. Top. Quantum Electron., 2014, 20, 1.

9 Y. Chen, G. Jiang, S. Chen, Z. Guo, X. Yu, C. Zhao and D. Fan, Mechanically exfoliated black phosphorus as a new saturable absorber for both Q-switching and mode-locking laser operation, Opt. Express, 2015, 23, 12823.

10 J. Lee, B. K. Yu, Y. I. Jhon, J. Koo, J. S. Kim, Y. M. Jhon and J. H. Lee, Filled skutterudites for broadband saturable absorbers, Adv. Opt. Mater., 2017, 5, 1700096.

11 Q. Bao, H. Zhang, Y. Wang, Z. Ni, Y. Yan, Z. X. Shen and D. Y. Tang, Atomic-layer graphene as a saturable absorber for ultrafast pulsed lasers, Adv. Funct. Mater., 2009, 19, 3077.

12 A. Martinez and Z. Sun, Nanotube and graphene saturable absorbers for fibre lasers, Nat. Photonics, 2013, 7, 842.

13 Z. Sun, T. Hasan, F. Torrisi, D. Popa, G. Privitera, F. Wang and A. C. Ferrari, Graphene mode-locked ultrafast laser, ACS Nano, 2010, 4, 803.

14 T. L. Feng, S. Z. Zhao, K. J. Yang, G. Q. Li, D. C. Li, J. Zhao and L. H. Zheng, Diode-pumped continuous wave tunable and graphene Q-switched Tm: LSO lasers. Optics express, Opt. Express, 2013, 21, 24665.

15 B. Fu, Y. Hua, X. Xiao, H. Zhu, Z. Sun and C. Yang, Broadband graphene saturable absorber for pulsed fiber lasers at 1, 1.5, and $2 \mu \mathrm{m}$, IEEE J. Sel. Top. Quantum Electron., 2014, 20, 411.

16 X. F. Yang, Y. Wang, H. T. Huang, D. Y. Shen, D. Y. Tang, H. Y. Zhu and J. Xu, A passively Q-switched Er: LuYAG laser with a graphene saturable absorber, Laser Phys. Lett., 2013, 10, 105810.

17 S. Wang, H. Yu, H. Zhang, A. Wang, M. Zhao, Y. Chen and J. Wang, Broadband Few-Layer $\mathrm{MoS}_{2}$ Saturable Absorbers, Adv. Mater., 2014, 26, 3538.
18 X. Wang, J. Xu, Z. You, Y. Sun, Z. Zhu and C. Tu, Triwavelength passively Q-switched $\mathrm{Yb}^{3+}: \mathrm{GdAl}_{3}\left(\mathrm{BO}_{3}\right)_{4}$ solidstate laser based on $\mathrm{WS}_{2}$ saturable absorber, Opt. Mater., 2016, 62, 621.

19 Q. H. Wang, K. Kalantar-Zadeh, A. Kis, J. N. Coleman and M. S. Strano, Electronics and optoelectronics of twodimensional transition metal dichalcogenides, Nat. Nanotechnol., 2012, 7, 699.

20 M. Thripuranthaka, R. V. Kashid, C. Sekhar Rout and D. J. Late, Temperature dependent Raman spectroscopy of chemically derived few layer $\mathrm{MoS}_{2}$ and $\mathrm{WS}_{2}$ nanosheets, Appl. Phys. Lett., 2014, 104, 081911.

21 D. Mao, Y. Wang, C. Ma, L. Han, B. Jiang, X. Gan and J. Zhao, $\mathrm{WS}_{2}$ mode-locked ultrafast fiber laser, Sci. Rep., 2015, 5, 7965.

22 Y. Tan, Z. Guo, L. Ma, H. Zhang, S. Akhmadaliev, S. Zhou and F. Chen, Q-Switched waveguide laser based on twodimensional semiconducting materials: tungsten disulfide and black phosphorous, Opt. Express, 2016, 24, 2858.

23 C. Luan, K. Yang, J. Zhao, S. Zhao, L. Song, T. Li and S. Jiang, $\mathrm{WS}_{2}$ as a saturable absorber for Q-switched 2 micron lasers, Opt. Lett., 2016, 41, 3783.

24 Y. Cheng, J. Peng, B. Xu, H. Yang, Z. Luo, H. Xu and J. Weng, Passive Q-Switching of a Diode-Pumped Pr: LiYF 4 Visible Laser Using $\mathrm{WS}_{2}$ as Saturable Absorber, IEEE Photonics J., 2016, 8, 1.

25 J. M. Serres, P. Loiko, X. Mateos, K. Yumashev, U. Griebner, V. Petrov and F. Díaz, Tm: $\mathrm{KLu}\left(\mathrm{WO}_{4}\right)_{2}$ microchip laser Qswitched by a graphene-based saturable absorber, Opt. Express, 2015, 23, 14108.

26 H. Huang, M. Li, P. Liu, L. Jin, H. Wang and D. Shen, Gold nanorods as the saturable absorber for a diode-pumped nanosecond Q-switched $2 \mu \mathrm{m}$ solid-state laser, Opt. Lett., 2016, 41, 2700.

27 Z. Chu, J. Liu, Z. Guo and H. Zhang, $2 \mu \mathrm{m}$ passively Qswitched laser based on black phosphorus, Opt. Mater. Express, 2016, 6, 2374.

28 P. Ge, J. Liu, S. Jiang, Y. Xu and B. Man, Compact Q-switched $2 \mu \mathrm{m}$ Tm: $\mathrm{GdVO}_{4}$ laser with $\mathrm{MoS}_{2}$ absorber, Photonics Res., 2015, 3, 256. 